\title{
Análisis Input-Output de la eficacia de la Política Europea de Desarrollo Rural 2007- 2013. Propuesta metodológica y resultados para Castilla-La Mancha
}

\section{LUÍS ANTONIO LÓPEZ}

Departamento de Análisis Económico y Finanzas, UNIVERSIDAD DE CASTILLA-LA MANCHA, ESPAÑA. E-mail: Luis.LSantiago@uclm.es

\section{FABIO MONSALVE}

Departamento de Análisis Económico y Finanzas, UNIVERSIDAD DE CASTILLA-LA MANCHA, ESPAÑA. E-mail: Fabio.Monsalve@uclm.es

\section{JORGE ENRIQUE ZAFRILLA}

Departamento de Análisis Económico y Finanzas, UNIVERSIDAD DE CASTILLA-LA MANCHA, ESPAÑA. E-mail: Jorge.Zafrilla@uclm.es

\section{RESUMEN}

La implementación del nuevo Fondo Europeo Agrícola de Desarrollo Rural (FEADER) refleja la decidida apuesta de la Unión Europea por convertir la Política de Desarrollo Rural en el segundo pilar de la Política Agrícola Común (PAC). La eficacia del FEADER para conseguir los objetivos propuestos será clave a la hora de determinar el éxito de esta nueva estrategia; de ahí la importancia de la evaluación de impacto. En este trabajo se propone una metodología, basada en el análisis input-output, que mediante un modelo multiregional permite evaluar los efectos sobre la producción, el valor añadido y empleo dentro de la región objetivo y fuera de ella (España, UE-27 y resto del mundo).

Palabras clave: Desarrollo Rural, Análisis Input-Output, Evaluación, Política Regional.

\section{The Effectiveness of European Rural Development Policy 2007- 2013. A Proposal for a Methodology on Input-Output Analysis and Empirical Evidence in the Case of Castilla - La Mancha}

\begin{abstract}
The new European Agricultural Fund for Rural Development (EAFRD) reflects the European Union firm support of the Rural Development Policy as the second pillar of the Common Agricultural Policy (CAP). The EAFRD effectiveness to attain the initial objectives will be the key aspect to the new strategy successfulness; hence the importance of impact evaluation. The paper proposed a Multi-regional Input-Output analysis (MRIO), which will allows us to evaluate the effects on aggregate production, value added and employment inside the Castilla-La Mancha region as well as outside (Spain, EU-27 and rest of the world).
\end{abstract}

Keywords: Rural development, Input-Ouput Analisys, Evaluation, Rural Policy.

Clasificación JEL: O18, R15

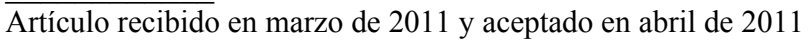

Artículo disponible en versión electrónica en la página www.revista-eea.net, ref. ə-29118 


\section{INTRODUCCIÓN}

El Reglamento 1290/2005 del Consejo de la Unión Europea sobre la financiación de la política agrícola común (PAC) ha supuesto un giro sustancial en el enfoque de intervención comunitaria en los espacios rurales. Mediante dicho Reglamento se han puesto en marcha "el Fondo Europeo Agrícola de Garantía (FEAGA), para financiar las medidas de mercado, y el Fondo Europeo Agrícola de Desarrollo Rural (FEADER), destinado a financiar los programas de desarrollo rural" (Comisión Europea, 2005a). Con la implementación de este segundo fondo, el desarrollo rural adquiere carta de naturaleza y se convierte finalmente en el demandado segundo pilar de la PAC (Colino y Martínez, 2005). Culmina así el largo camino iniciado con el seminal documento "El futuro del mundo rural" (Comisión Europea, 1989) en el que, frente a la visión productivista de la PAC, la Comisión apostaba por una nueva metodología de intervención caracterizada por enfatizar el desarrollo no agrario de las zonas rurales (visión plurisectorial), la protección medioambiental (sostenibilidad), la valorización de los recursos físicos y humanos y la implicación de la población local (carácter endógeno).

El éxito y pervivencia de esta nueva política dependerá de la eficacia del FEADER para conseguir los objetivos propuestos, lo que convierte la evaluación en una pieza clave del sistema; evaluación, por otra parte, de especial complejidad debido a la mezcla de elementos cuantitativos y, sobre todo, cualitativos (Ray, 2000) ${ }^{1}$.

En sentido estricto, el FEADER tiene por objetivo la "promoción de un desarrollo rural sostenible" mediante una metodología de intervención particular basada en los modelos de desarrollo endógeno (Comisión Europea, 2005b). Ahora bien, es previsible que el impacto de las importantes dotaciones presupuestarias que se van a inyectar en las regiones objetivo sobrepase dichas regiones e incluso el país. En este sentido, puede resultar interesante analizar el efecto arrastre de dichos fondos europeos tanto sobre el territorio como fuera de él. Para ello la metodología input-output resulta especialmente indicada.

El método input-output se ha utilizado con anterioridad para medir el impacto económico de los fondos europeos si bien, en la mayoría de los casos, se ha tenido en cuenta el montante global de la intervención (Fondos Estructurales y de Cohesión) y no específicamente los fondos de desarrollo rural (Fondo Europeo de Orientación y Garantía Agrícola-Sección Orientación y

\footnotetext{
${ }^{1}$ El FEADER ha de contribuir a alcanzar los siguientes objetivos (Comisión Europea, 2005b, art. 4): a) Aumentar la competitividad de la agricultura y la silvicultura mediante la ayuda a la reestructuración, el desarrollo y la innovación; b) Mejorar el medio ambiente y el medio rural mediante ayudas a la gestión de tierras; c) Mejorar la calidad de vida en las zonas rurales y fomentar la diversificación de la actividad económica.
} 
LEADER). Los modelos de análisis más comunes han sido: a) Los estudios a través de la elaboración de matrices de contabilidad social, como el de Cámara y Marcos (2009) para la Comunidad de Madrid 2000-2006 y el de Lima y Cardenete (2005), para la Comunidad de Andalucía 1989-2005; estas matrices también se han utilizado para realizar estudios basados en modelos regionales de equilibrio general aplicado regional, como los recogidos en (Cardenete y Llop, 2005); b) Los modelos uniregionales, como los de Marín y Pardo (2009) para la Comunidad de Castilla y León 2000-2006 y el de Murillo (2007) para la Comunidad de Andalucía; c) Los modelos multiregionales, como el de Llano (2004) que trata de medir la importancia de la proximidad geográfica y la especialización sectorial en las relaciones comerciales para las 17 comunidades autónomas y el de Pérez, Dones y Llano (2009) que, considerando las 17 comunidades autónomas más Ceuta y Melilla, han tratado de medir los efectos interregionales e intersectoriales de los Fondos Estructurales (1995-1999) sobre la producción, el valor añadido y el empleo. La conclusión de este último estudio es significativa: el impacto de los fondos recibidos es mayor en términos relativos en las regiones que son más ricas, las objetivo 2 frente a las objetivo 1, ya que parte del impacto sobre las regiones más pobres se desvía hacia las otras a través de la compra de mercancías finales e intermedias a estas últimas.

El presente trabajo se engloba dentro de este último enfoque. Para realizar el estudio se va a plantear un modelo input-output multiregional que permitirá calcular los impactos de los programas de desarrollo rural sobre la producción, el valor añadido y el empleo tanto dentro de la región objetivo (Castilla-La Mancha) como fuera de ella (España, UE-27 y resto del mundo). Esto último permitirá tener en cuenta los impactos sobre el comercio internacional, identificando no sólo qué parte del impacto se transfiere a otras regiones, sino también qué parte va fuera de la Unión Europea.

Adicionalmente el trabajo plantea la evaluación de impacto diferenciando entre fondos destinados a las transferencias directas de rentas a la población y fondos destinados a financiar las iniciativas y proyectos de desarrollo rural propiamente dichos. Teniendo en cuenta que la demanda final de la región se divide entre consumo final de los hogares, inversión y exportaciones, las transferencias directas de rentas van a implicar un aumento del consumo final de las familias (y por tanto de la demanda final), mientras que las ayudas financieras a los distintos proyectos van a suponer un aumento del conjunto de la demanda final de la región. La metodología propuesta permitirá evaluar qué tipo de fondos va a tener un mayor impacto, tanto en Castilla-La Mancha como fuera de ella.

En el apartado siguiente se presenta brevemente el Programa de Desarrollo Rural de Castilla-La Mancha. En el tercer epígrafe se describe la metodología 
empleada. En el cuarto se describen y analizan los resultados obtenidos de la evaluación de impacto. Finalmente, en el quinto y último apartado, se recogen las conclusiones del estudio.

\section{EL PROGRAMA DE DESARROLLO RURAL DE CASTILLA- LA MANCHA 2007-2013}

El Programa de Desarrollo Rural de Castilla-La Mancha $2007-2013^{2}$ es el documento que recoge la estrategia, medidas de intervención y propuesta de financiación, mediante el cual la Comunidad Autónoma solicitó las ayudas del Fondo Europeo Agrícola de Desarrollo Rural (FEADER) (Junta de Comunidades de Castilla-la Mancha, 2009). Las actuaciones recogidas en dicho documento se articulan en torno a los 4 ejes de intervención contemplados por el FEADER. Veamos brevemente en qué consisten:

Eje 1. Aumento de la competitividad del sector agrícola y forestal. En este eje se han incluido 12 medidas con el doble objetivo de fomentar el conocimiento y mejora el potencial humano de las explotaciones agrarias y reestructurar e impulsar las explotaciones agrarias para mejorar el capital físico así como la calidad de la producción.

Eje 2. Mejora del medio ambiente y del entorno rural. Este eje comprende 6 medidas destinadas a la utilización sostenible de las tierras agrícolas y forestales.

Eje 3. Mejora de la calidad de vida en las zonas rurales y fomento de la diversificación de la actividad económica. En este eje se contemplan 8 medidas con la prioridad principal de la creación y mantenimiento de empleo en las zonas rurales por medio del fomento de nuevas actividades económicas.

Eje 4. LEADER. En este eje se incluyen medidas que contribuyen a la consecución de los objetivos del eje 3, pero aplicadas siguiendo la metodología LEADER; es decir, mediante estrategias de desarrollo territoriales, endógenas y gestionadas por la propia población a través de los grupos de acción local.

El total de gasto público contemplado para la ejecución de dichas medidas es de 1.852.084.985 euros, de los cuales 1.133.445.363 los aporta la Unión Europea a través del FEADER, 407.977.883 la Administración General del Estado y 310.661.739 la Administración Regional. Se prevé que dicho gasto público genere, al menos, un gasto privado de 1.481.831.292 euros. Es decir, si el cuadro financiero aprobado se ejecuta en su totalidad, la aplicación del

${ }^{2}$ El Programa de Desarrollo Rural de Castilla-La Mancha fue aprobado por Decisión de la Comisión Europea C(2008) 3832, de 16 de julio y modificado por la Decisión C(2010) 1229, de 8 de marzo de 2010. 
FEADER movilizará, como mínimo, más de 3.200 millones de euros en Castilla-La Mancha durante el septenio 2007-2013. La Tabla 1 recoge la distribución de los fondos por ejes-medidas y por fuentes de financiación.

Tabla 1

Cuadro Financiero del Programa de Desarrollo Rural de Castilla-La Mancha 2007-2013

\begin{tabular}{|c|c|c|c|c|c|c|}
\hline Medida & $\begin{array}{c}\text { Gasto } \\
\text { Público }\end{array}$ & FEADER & AGE & Regional & $\begin{array}{c}\text { Gasto } \\
\text { Privado }\end{array}$ & Total \\
\hline $\begin{array}{l}\text { Medida 111: Acciones relativas a la } \\
\text { información y la formación profesional }\end{array}$ & 8.100 .000 & 5.210 .000 & 2.486 .000 & 404.000 & 0 & 8.100 .000 \\
\hline $\begin{array}{l}\text { Medida 112: Instalación de jóvenes } \\
\text { agricultores }\end{array}$ & 82.000 .000 & 52.480 .000 & 14.760 .000 & 14.760 .000 & 82.000 .000 & 164.000 .000 \\
\hline $\begin{array}{l}\text { Medida 113: Jubilación anticipada de } \\
\text { los agricultores y trabajadores agrícolas }\end{array}$ & 39.000 .000 & 24.960 .000 & 12.090 .000 & 1.950 .000 & 0 & 39.000 .000 \\
\hline $\begin{array}{l}\text { Medida 114: Utilización de servicios de } \\
\text { asesoramiento }\end{array}$ & 17.500 .000 & 11.304 .000 & 3.102 .000 & 3.094 .000 & 4.375 .000 & 21.875 .000 \\
\hline $\begin{array}{l}\text { Medida 115: Implantación de servicios } \\
\text { de asesoramiento a las explotaciones } \\
\text { agrícolas y forestales }\end{array}$ & 3.000 .000 & 2.310 .000 & 360.000 & 330.000 & 1.300 .000 & 4.300 .000 \\
\hline $\begin{array}{l}\text { Medida 121: Modernización de las } \\
\text { explotaciones agrícolas }\end{array}$ & 101.650 .000 & 67.864 .000 & 28.811 .500 & 4.974 .500 & 110.475 .000 & 212.125 .000 \\
\hline $\begin{array}{l}\text { Medida 123: Aumento del valor añadido } \\
\text { de los productos agrícolas y forestales }\end{array}$ & 294.650 .127 & 190.448 .081 & 52.173 .023 & 52.029 .023 & 1.092 .800 .000 & 1.387 .450 .127 \\
\hline $\begin{array}{l}\text { Medida 124: Cooperación para el } \\
\text { desarrollo de nuevos productos, } \\
\text { procesos y tecnologías en el sector } \\
\text { agrícola y alimentario y en el sector } \\
\text { forestal }\end{array}$ & 5.000 .000 & 3.200 .000 & 1.550 .000 & 250.000 & 1.700 .000 & 6.700 .000 \\
\hline $\begin{array}{l}\text { Medida 125: Infraestructuras } \\
\text { relacionadas con la evolución y } \\
\text { adaptación de la agricultura y la } \\
\text { silvicultura }\end{array}$ & 111.535 .000 & 72.812 .400 & 19.416 .300 & 19.306 .300 & 56.535 .000 & 168.070 .000 \\
\hline $\begin{array}{l}\text { Medida 126: Reconstitución del } \\
\text { potencial de producción agrícola } \\
\text { dañado por catástrofes naturales y la } \\
\text { implantación de medidas preventivas } \\
\text { adecuadas }\end{array}$ & 1.994 .559 & 1.276 .518 & 0 & 718.041 & 0 & 1.994 .559 \\
\hline $\begin{array}{l}\text { Medida 132: Participación de los } \\
\text { agricultores en programas relativos a la } \\
\text { calidad de los alimentos }\end{array}$ & 17.080 .000 & 10.931 .200 & 5.294 .800 & 854.000 & 18.584 .300 & 35.664 .300 \\
\hline $\begin{array}{l}\text { Medida 133: Apoyo a las agrupaciones } \\
\text { de productores en actividades de } \\
\text { información y promoción }\end{array}$ & 7.000 .000 & 4.480 .000 & 2.170 .000 & 350.000 & 3.000 .000 & 10.000 .000 \\
\hline Total EJE 1 & 688.509 .686 & 447.276.199 & 142.213 .623 & 99.019 .864 & 1.370 .769 .300 & 2.059.278.986 \\
\hline $\begin{array}{l}\text { Medida 211: Ayudas destinadas a } \\
\text { indemnizar a los agricultores por las } \\
\text { dificultades naturales en las zonas de } \\
\text { montaña }\end{array}$ & 92.350 .000 & 51.368 .838 & 24.785 .263 & 16.195 .899 & 0 & 92.350 .000 \\
\hline $\begin{array}{l}\text { Medida 212: Ayudas destinadas a } \\
\text { indemnizar a los agricultores por las } \\
\text { dificultades naturales en las zonas } \\
\text { distintas de las de montaña }\end{array}$ & 45.300 .000 & 23.360 .214 & 13.142.617 & 8.797 .169 & 0 & 45.300 .000 \\
\hline Medida 214: Ayudas agroambientales & 474.950 .073 & 276.568 .522 & 119.554 .044 & 78.827 .507 & 0 & 474.950 .073 \\
\hline $\begin{array}{l}\text { Medida 221: Primera forestación de } \\
\text { tierras agrícolas }\end{array}$ & 171.000 .000 & 90.784 .921 & 48.213 .944 & 32.001 .135 & 5.000 .000 & 176.000 .000 \\
\hline $\begin{array}{l}\text { Medida 226: Ayudas a la recuperación } \\
\text { del potencial forestal e implantación de } \\
\text { medidas preventivas }\end{array}$ & 107.000 .004 & 60.187 .502 & 26.427 .500 & 20.385 .002 & 0 & 107.000 .004 \\
\hline Medida 227: Inversiones no productivas & 90.479 .200 & 50.325 .861 & 8.302 .948 & 31.850 .391 & 090.479 .200 & \\
\hline Total EJE 2 & 981.079.277 & 552.595 .858 & 240.426 .316 & 188.057.103 & 5000000 & 895.600 .077 \\
\hline
\end{tabular}


Tabla 1 (continuación)

Cuadro Financiero del Programa de Desarrollo Rural de Castilla-La Mancha 2007-2013

\begin{tabular}{|c|c|c|c|c|c|c|}
\hline Medida & $\begin{array}{c}\text { Gasto } \\
\text { Público }\end{array}$ & FEADER & AGE & Regional & $\begin{array}{l}\text { Gasto } \\
\text { Privado }\end{array}$ & Total \\
\hline $\begin{array}{l}\text { Medida 313.2: Fomento de actividades } \\
\text { turísticas }\end{array}$ & 4.261 .992 & 3.153 .874 & 596.679 & 511.439 & 4.261 .992 & 8.523 .984 \\
\hline $\begin{array}{l}\text { Medida } 321.2 \text { : Servicios básicos para la } \\
\text { economía y la población rural }\end{array}$ & 15.000 .000 & 11.100 .000 & 2.100 .000 & 1.800 .000 & 0 & 15.000 .000 \\
\hline $\begin{array}{l}\text { Medida 323.2: Conservación y mejora } \\
\text { del patrimônio natural }\end{array}$ & 5.400 .004 & 3.996 .003 & 756.001 & 648.000 & 0 & 5.400 .004 \\
\hline Total EJE 3 & 24.661.996 & 18.249.877 & 3.452 .680 & 2.959 .439 & 4.261.992 & 28.923.988 \\
\hline $\begin{array}{l}\text { Medida 413: Calidad de } \\
\text { vida/diversificación }\end{array}$ & 119.000 .000 & 88.060 .000 & 16.660 .000 & 14.280 .000 & 101.800 .000 & 220.800 .000 \\
\hline Medida 421: Cooperación & 3.667 .222 & 2.713 .744 & 513.411 & 440.067 & 0 & 3.667 .222 \\
\hline $\begin{array}{l}\text { Medida 431: Costes de funcionamiento, } \\
\text { adquisición de competencias,promoción }\end{array}$ & 30.666 .804 & 22.693 .435 & 4.293 .353 & 3.680 .016 & 0 & 30.666 .804 \\
\hline Total EJE 4 & 153.334 .026 & 113.467 .179 & 21.466.764 & 18.400 .083 & 101.800 .000 & 255.134 .026 \\
\hline Medida 511: Asistencia técnica & 4.500 .000 & 156.250 & 418.500 & 2.225 .250 & 0 & 4.500 .000 \\
\hline TOTAL GENERAL & 1.852 .084 .985 & 1.131 .745 .363 & 407.977 .883 & 310.661 .739 & 1.481 .831 .292 & 3.243.437.077 \\
\hline
\end{tabular}

\section{PLANTEAMIENTO METODOLOGICO}

\subsection{Descripción del modelo}

El modelo propuesto es un modelo input-output multiregional (MRIO) con comercio unidireccional. Su principal salida es el nivel de producción asociada a la demanda final de la región 1, que será la región objetivo de Castilla-La Mancha al objeto del presente estudio (Andrew, Peters y Lennox, 2009). Debido a la limitación de los datos disponibles, el modelo contempla únicamente un comercio unidireccional; es decir, la región 1 comercia con el resto de regiones, mientras que el resto no comercian entre ellas ${ }^{3}$. La expresión matemática que considera el total de producción de las distintas ramas de una región $1\left(\mathrm{x}_{1}\right)$ más la producción realizada por el resto de regiones $\left(\mathrm{x}_{2}, \ldots, \mathrm{x}_{\mathrm{m}}\right)$ para satisfacer la demanda final de la región 1 sería la siguiente:

$$
\left(\begin{array}{c}
\mathrm{x}_{1} \\
\mathrm{x}_{2} \\
\mathrm{x}_{3} \\
\vdots \\
\mathrm{x}_{\mathrm{r}}
\end{array}\right)=\left(\begin{array}{ccccc}
\mathrm{A}_{11} & 0 & 0 & \cdots & 0 \\
\mathrm{~A}_{21} & \mathrm{~A}_{2} & 0 & \cdots & 0 \\
\mathrm{~A}_{31} & 0 & \mathrm{~A}_{3} & \cdots & 0 \\
\vdots & \vdots & \vdots & \ddots & \vdots \\
\mathrm{A}_{\mathrm{r} 1} & 0 & 0 & \cdots & \mathrm{A}_{\mathrm{r}}
\end{array}\right)\left(\begin{array}{c}
\mathrm{x}_{1} \\
\mathrm{x}_{2} \\
\mathrm{x}_{3} \\
\vdots \\
\mathrm{x}_{\mathrm{r}}
\end{array}\right)+\left(\begin{array}{c}
\mathrm{y}_{11} \\
\mathrm{y}_{21} \\
\mathrm{y}_{31} \\
\vdots \\
\mathrm{y}_{\mathrm{r} 1}
\end{array}\right)
$$

${ }^{3}$ La tabla Input-output de Castilla-La Mancha nos informa del comercio de esa región con España, la Unión Europea y el resto del mundo. Sin embargo, las tablas input-output utilizadas en el trabajo para reflejar la tecnología de esas regiones no recogen el comercio entre ellas (véase, la sección 3.3 para un mayor detalle). 
En donde, $\mathrm{A}_{11}$ es la matriz de coeficientes técnicos domésticos de la región $1, A_{21}, \ldots, A_{r 1}$, son las matrices de coeficientes técnicos importados de la región 1 del resto de regiones. En relación con $A_{2}, \ldots, A_{r}$ cabe señalar que son las matrices de coeficientes técnicos totales de esas regiones, es decir la suma de la matriz doméstica más la importada; de esta manera se incluyen todas las rondas necesarias para la producción de las mercancías. Implícitamente estamos suponiendo que las importaciones que realizan esos países se realizan con la misma tecnología que la producción doméstica ${ }^{4}$. El vector $\mathrm{y}_{11}$ recoge la demanda final (consumo, inversión y exportaciones) de la región 1 por ramas de actividad y el resto de vectores $\mathrm{y}_{21}, \mathrm{y}_{31}, \ldots, \mathrm{y}_{\mathrm{r} 1}$ consideran las importaciones de demanda final que realiza el región 1 al resto de regiones.

Debido a que la matriz fuera de la diagonal es un bloque de ceros, la resolución de este sistema de ecuaciones puede simplificarse separando los cálculos de cada región de análisis (Peters y Hertwich, 2006). El resultado viene dado por la ecuación (2) que refleja la producción de la región 1 asociada a su demanda final y la ecuación (3) que cuantifica la producción de bienes intermedios y de bienes finales de una región $\mathrm{r}$ necesaria para satisfacer la demanda de la región 1.

$$
\begin{aligned}
& \mathrm{x}_{1}=\left(1-\mathrm{A}_{11}\right)^{-1} \mathrm{y}_{11} \\
& \mathrm{x}_{\mathrm{r}}=\left(1-\mathrm{A}_{\mathrm{r}}\right)^{-1}\left(\mathrm{~A}_{\mathrm{r} 1}\left(1-\mathrm{A}_{11}\right)^{-1} \mathrm{y}_{11}+\mathrm{y}_{\mathrm{r} 1}\right)
\end{aligned}
$$

En definitiva, el modelo propuesto permite evaluar el impacto del Programa de Desarrollo Rural sobre la producción tanto de la región objetivo como de las regiones con las que ésta comercia. Asimismo, pueden fácilmente obtenerse también los efectos sobre el valor añadido y el empleo. En el siguiente apartado se explica la metodología de estos cálculos.

\subsection{El Análisis de impacto del Programa de Desarrollo Rural}

El primer paso para medir los efectos del Programa de Desarrollo Rural consiste en definir la nueva demanda final incrementada, mediante el cual se podrá, en una fase ulterior, calcular el impacto sobre la producción, el valor añadido y el empleo tanto en la región objetivo como en el resto de regiones.

\footnotetext{
${ }^{4} \mathrm{Al}$ hacer estos supuestos, los datos mostrarán unos efectos mayores a los que realmente se dan en la economía española, en la Unión Europea y en el Resto del Mundo, pues asumimos que estas tres regiones atienden toda la demanda generada por la implantación de los Programas de Desarrollo Rural con producción interior, sin recurrir a la importación (supuesto no realista). Por tanto asumimos que no derivan parte del efecto multiplicador al extranjero, que es lo que en realidad estaría pasando. A pesar de este inconveniente, el estudio nos permite aproximar la magnitud del impacto fuera de Castilla-La Mancha de los Programas de Desarrollo Rural bajo los supuestos establecidos.
} 
Para construir dicho vector, al objeto del presente estudio, los fondos recibidos del FEADER van a desagregarse en dos tipos; por una parte, se consideran las ayudas destinadas a mantener la renta de los agricultores y que se formalizan en transferencias directas; por otra, las destinadas a la ejecución de los distintos proyectos de inversión. Ambos tipos de ayudas generan un aumento de la demanda final en la región objetivo, aunque con diferentes características. Mientras que las ayudas a rentas se destinan totalmente a consumo $(\Delta \mathrm{rc})^{5}$, las ayudas a proyectos suponen un aumento del conjunto de la demanda final $(\Delta \mathrm{d})$.

Una vez obtenidos los dos nuevos vectores incrementados con las ayudas, el siguiente paso en el análisis consiste en dividir el consumo, entre doméstico e importado. Para ello se utiliza la información suministrada por el vector de consumo de la tabla input-output de la región de Castilla-La Mancha.

En relación con las ayudas a rentas, para calcular el nuevo vector de consumo se ha procedido a dividir el conjunto de las ayudas presupuestadas por la proporción que, sobre el total, supone el consumo de cada una de las ramas. Asimismo se ha procedido a diferenciar entre el consumo de bienes domésticos de la región $1\left(\Delta \mathrm{rc}_{11}\right)$ y el consumo de bienes importados de las restantes $\mathrm{r}$ regiones consideradas $\left(\Delta \mathrm{rc}_{\mathrm{r} 1}\right)$. El vector resultante es el siguiente:

$$
\Delta \mathrm{rc}=\left(\begin{array}{c}
\Delta \mathrm{rc}_{11} \\
\Delta \mathrm{rc}_{21} \\
\Delta \mathrm{rc}_{31} \\
\vdots \\
\Delta \mathrm{rc}_{\mathrm{r} 1}
\end{array}\right)
$$

Por lo que se refiere a las ayudas a proyectos, para calcular el nuevo vector de demanda final se ha procedido a diferenciar entre el aumento de la demanda doméstica $\left(\Delta \mathrm{d}_{11}\right)$ y el aumento de la demanda importada de las $\mathrm{r}$ regiones consideradas $\left(\Delta \mathrm{d}_{\mathrm{r} 1}\right)$; tomando como criterio de asignación la proporción en la cual la demanda final (consumo, inversión y exportaciones) se distribuye entre ambos tipos de consumo, doméstico e importado según se recoge en la tabla input-output de la región objetivo ${ }^{6}$. El nuevo vector obtenido sería el siguiente:

\footnotetext{
${ }^{5}$ Suponemos una nula propensión al ahorro.

${ }^{6}$ La distribución propuesta entre demanda doméstica e importada de las ayudas a rentas es, por tanto, distinta a la que se propone para las ayudas a proyectos. Mientras que la renta se distribuye en función de los gastos de consumo que se hace en la economía nacional, la segunda se distribuye en función de la demanda final, parte de la cual se destina a gastos de inversión.
} 


$$
\Delta \mathrm{d}=\left(\begin{array}{c}
\Delta \mathrm{d}_{11} \\
\Delta \mathrm{d}_{21} \\
\Delta \mathrm{d}_{31} \\
\vdots \\
\Delta \mathrm{d}_{\mathrm{r} 1}
\end{array}\right)
$$

Si sustituimos el vector de demanda final de la expresión (1), por los vectores (4) y (5) podemos calcular el efecto sobre la producción que el Programa de Desarrollo Rural tendrá en todas las regiones consideradas. En concreto, el impacto sobre la producción doméstica de la región objetivo vendrá determinado por las siguientes expresiones:

$$
\begin{aligned}
& \Delta \mathrm{x}_{1}^{\mathrm{rc}}=\left(1-\mathrm{A}_{11}\right)^{-1} \Delta \mathrm{rc}_{11} \\
& \Delta \mathrm{x}_{1}^{\mathrm{d}}=\left(1-\mathrm{A}_{11}\right)^{-1} \Delta \mathrm{d}_{11}
\end{aligned}
$$

La expresión (6) cuantifica el aumento la producción dentro de la región objetivo vinculado al crecimiento de las ayudas a rentas que suponen esos fondos y la (7) el aumento de la producción dentro de esa región derivada de las ayudas a proyectos.

Una vez obtenidos los vectores incrementados de consumo y demanda final es relativamente sencillo calcular los efectos sobre el valor añadido y el empleo. Definimos los vectores $\mathrm{v}_{1}=\mathrm{va} / \mathrm{x}_{1}$ que cuantifica el valor añadido (va) por unidad producida (x) y el coeficiente laboral $\mathrm{e}_{1}=\mathrm{l}_{1} / \mathrm{x}_{1}$ que mide el empleo (l) por unidad producida. A partir de aquí, las siguientes expresiones permiten calcular el valor añadido $\left(\Delta \mathrm{va}_{1}{ }^{\mathrm{d}}\right.$ y $\left.\Delta \mathrm{va}_{1}{ }^{\mathrm{rc}}\right)$ y el empleo asociado $\left(\Delta \mathrm{l}_{1}{ }^{\mathrm{d}}\right.$ y $\left.\Delta \mathrm{l}_{1}{ }^{\mathrm{rc}}\right)$ a los fondos FEADER $^{7}$ :

$$
\begin{aligned}
& \Delta \mathrm{va}_{1}^{\mathrm{rc}}=\mathrm{v}_{1}\left(1-\mathrm{A}_{11}\right)^{-1} \Delta \mathrm{rc}_{11} \\
& \Delta \mathrm{va}_{1}^{\mathrm{d}}=\mathrm{v}_{1}\left(1-\mathrm{A}_{11}\right)^{-1} \Delta \mathrm{d}_{11} \\
& \Delta \mathrm{l}_{1}^{\mathrm{rc}}=\mathrm{e}_{1}\left(1-\mathrm{A}_{11}\right)^{-1} \Delta \mathrm{rc}_{11} \\
& \Delta \mathrm{l}_{1}^{\mathrm{d}}=\mathrm{e}_{1}\left(1-\mathrm{A}_{11}\right)^{-1} \Delta \mathrm{d}_{11}
\end{aligned}
$$

${ }^{7}$ Otra posibilidad adicional es diagonalizar los vectores de demanda final de tal forma que podamos diferenciar entre los efectos directos que sobre el empleo tienen los dos vectores demanda final y los efectos indirectos, aquellos que se producen por arrastrar del resto de ramas de la economía. 
Como ya se ha señalado, el impacto de los programas de desarrollo rural no se restringe al ámbito de la región objetivo, sino que parte se transfiere al resto de economías con las que comercia, especialmente a través de las importaciones de bienes finales e intermedios necesarias para satisfacer el crecimiento de la demanda final.

Las importaciones directas e indirectas de bienes intermedios procedentes de la región $r$ y necesarias para satisfacer los aumentos de demanda como consecuencia del Programa de Desarrollo Rural, vienen dados, respectivamente, por las expresiones (12) y (13).

$$
\begin{aligned}
& \Delta \mathrm{m}_{\mathrm{r}}^{\mathrm{rc}}=\mathrm{A}_{\mathrm{r} 1}\left(1-\mathrm{A}_{11}\right)^{-1} \Delta \mathrm{rc}_{11} \\
& \Delta \mathrm{m}_{\mathrm{r}}^{\mathrm{d}}=\mathrm{A}_{\mathrm{r} 1}\left(1-\mathrm{A}_{11}\right)^{-1} \Delta \mathrm{d}_{11}
\end{aligned}
$$

Las importaciones totales $\left(\Delta \mathrm{m}_{\mathrm{r}}^{\mathrm{T}}\right)$ de cada región, que incluyen las de bienes de consumo y de bienes intermedios asociadas al Programa serán:

$$
\Delta \mathrm{m}_{\mathrm{r}}^{\mathrm{T}}=\sum_{\mathrm{r}=2}^{\mathrm{m}} \Delta \mathrm{d}_{\mathrm{r} 1}+\sum_{\mathrm{r}=2}^{\mathrm{m}} \Delta \mathrm{rc}_{\mathrm{r} 1}+\sum_{\mathrm{r}=2}^{\mathrm{m}} \Delta \mathrm{m}_{\mathrm{r}}^{\mathrm{d}}+\sum_{\mathrm{r}=2}^{\mathrm{m}} \Delta \mathrm{m}_{\mathrm{r}}^{\mathrm{rc}}
$$

Finalmente, una vez que se dispone del total de importaciones asociadas al programa de desarrollo en la región objetivo se proponen calcular los efectos totales, sobre la producción (15), el valor añadido (16) y el empleo (17) de las $r$ economías que suministran esas mercancías. Siendo, además, $\mathrm{v}_{\mathrm{j}}$ el coeficiente de valor añadido y $e_{j}$ el coeficiente de empleo de la región j.

$$
\begin{aligned}
& \Delta \mathrm{x}_{\mathrm{r}}=\left(1-\mathrm{A}_{\mathrm{r}}\right)^{-1} \Delta \mathrm{m}_{\mathrm{r}}^{\mathrm{T}} \\
& \Delta \mathrm{va}_{\mathrm{r}}=\mathrm{v}_{\mathrm{r}}\left(1-\mathrm{A}_{\mathrm{r}}\right)^{-1} \Delta \mathrm{m}_{\mathrm{r}}^{\mathrm{T}} \\
& \Delta \mathrm{l}_{\mathrm{r}}=\mathrm{e}_{\mathrm{r}}\left(1-\mathrm{A}_{\mathrm{r}}\right)^{-1} \Delta \mathrm{m}_{\mathrm{r}}^{\mathrm{T}}
\end{aligned}
$$

\subsection{Las fuentes de datos}

Las tabla input-output simétrica utilizada para la región objetivo es la correspondiente al año 2005, última publicada por el Instituto de Estadística de Castilla-La Mancha; este marco input-output diferencia entre las relaciones domésticas e importadas procedentes de España, la Unión Europea y el resto del mundo. A fin de garantizar la coherencia temporal, las tablas consideradas para el resto de regiones son también de 2005. Para el caso de la tabla simétrica de España, se han utilizado los datos de la tabla total (doméstica e importada) elaborada por el Instituto Nacional de Estadística. Para los países de la Unión Europea y del resto del mundo se han usado las tablas input-output totales 
suministradas por la base de datos de la OCDE. Los países seleccionados para construir la matriz de la Unión Europea han sido aquellos para los que se disponía información para el año de referencia, 2005: Alemania, Francia, Reino Unido, Italia, Portugal, Grecia, Irlanda y Luxemburgo; estos países absorben el $74 \%$ de las importaciones procedentes de la Unión Europea que realizó la Comunidad de Castilla-La Mancha durante el año 2005. En la región resto del mundo se han incluido EEUU, China y Japón; estos tres países suministran, por sí solos, el $48 \%$ del total de las importaciones de Castilla-La Mancha ${ }^{8}$.

El desfase temporal entre los datos input-output utilizados (2005) y los años en los que el FEADER se va a ejecutar (2007-2013) ${ }^{9}$ supone una limitación al modelo que debemos tener en cuenta. Sin embargo, este desfase es común a la hora de trabajar con la metodología input-output debido, principalmente, a las dificultades y a la lentitud que presenta la elaboración de estas tablas por parte de los organismos competentes. Independientemente de lo anterior, conviene tener presente que el impacto de los fondos puede verse afectado por los cambios en las relaciones intersectoriales que se hayan producido durante el periodo de estudio; cambios que pueden ser más significativos debido a la crisis económica.

\section{Tabla 2}

Agregación Tabla Input-Output Castilla-La Mancha de 62 ramas a $16\left(\mathrm{TIO}_{16}\right)$

\begin{tabular}{|c|l|c|}
\hline \multicolumn{1}{|c|}{ Ramas TIO (16) 2005 } & $\begin{array}{c}\text { Correspondencias Ramas } \\
\text { TIO (62) 2005 }\end{array}$ \\
\hline 1 & Agricultura, Ganadería, caza, selvicultura y pesca & $1-2$ \\
\hline 2 & Industrias Extractivas & 3 \\
\hline 3 & Energía y Agua & $4-5$ \\
\hline 4 & Industria mafucturera & $6-27 ; 32-33$ \\
\hline 5 & Maquinaria y material de transporte & $28-31$ \\
\hline 6 & Construcción & $34-35$ \\
\hline 7 & Comercio y reparación & $36-39$ \\
\hline
\end{tabular}

\footnotetext{
${ }^{8}$ Toda la información ha sido agregada a 16 ramas de actividad homogéneas. La tabla de CastillaLa Mancha dispone de 62 ramas, la de España cuenta con 73 y la del resto de países con un total de 48. Además, todos los datos están expresados en euros, por lo que la información de Reino Unido, EEUU, China y Japón ha sido convertida a euros.

${ }^{9}$ Los datos han sido deflactados utilizando el IPC de Castilla-La Mancha para los fondos destinados a rentas y los índices de volumen encadenados, suministrados por la Contabilidad Regional, para los fondos asignados a proyectos. En este último caso, dada la disponibilidad de datos, se han usado los índices encadenados correspondientes de la agricultura y de la construcción y, para el resto de ramas, el del PIB. La información sobre ejecución del gasto sólo estaba disponible hasta el año 2010. Para años posteriores se han usado los deflactores e índices encadenados del último año disponible: 2010.
} 
Tabla 2 (continuación)

Agregación Tabla Input-Output Castilla-La Mancha de 62 ramas a $16\left(\mathrm{TIO}_{16}\right)$

\begin{tabular}{|c|l|c|}
\hline \multicolumn{1}{|c|}{ Ramas TIO (16) 2005 } & $\begin{array}{c}\text { Correspondencias Ramas } \\
\text { TIO (62) 2005 }\end{array}$ \\
\hline 8 & Hostelería & $40-41$ \\
\hline 9 & Transportes y comunicaciones & $42-46$ \\
\hline 10 & Intermediación financiera & $47-49$ \\
\hline 11 & Servicios prestados a las empresas & $50-53$ \\
\hline 12 & Administración pública & $54.1-54.3$ \\
\hline 13 & Educación & $55.1-55.2$ \\
\hline 14 & Sanidad y servicios sociales & $56.1-57.2$ \\
\hline 15 & Otras actividades sociales y servicios personales & $58-61$ \\
\hline 16 & Actividades de los hogares & 62 \\
\hline
\end{tabular}

En este punto, cabe resaltar también la existencia de otros condicionantes que presenta el análisis input-output, los cuales han de ser identificados a la hora de llevar a cabo este tipo de estudios. Estos son las asunciones de proporcionalidad y homogeneidad (Acquaye y Duffy, 2010). El supuesto de proporcionalidad refleja que cuando el output de un sector se incrementa también lo hace, proporcionalmente, la demanda de bienes intermedios, no existiendo economías de escala. El supuesto de homogeneidad implica que la agregación de las ramas puede conllevar un reducido grado de refinamiento, con lo que la estructura productiva de determinadas industrias podría no ser la adecuada de cara a recoger todas las interconexiones sectoriales. El reducido grado de agregación sectorial que presenta nuestro modelo, detallado en la Tabla 2, supone, en línea con el supuesto de homogeneidad, una limitación adicional que debemos tener en cuenta a la hora de interpretar los resultados.

\subsection{La regla de reparto}

El reparto de los fondos para la realización del presente estudio se ha efectuado desde una doble perspectiva. La primera, es la ya mencionada diferenciación entre fondos previsiblemente destinados a ayudas a rentas (aumento consumo- $\Delta r c$ ) y fondos destinados a proyectos de inversión (aumento de la demanda final- $\Delta d$ ). En concreto, el 42,94\% (795 millones de euros) del total del gasto público se ha preasignado como ayudas a rentas y el $57,06 \%$ (1.056 millones de euros) se ha considerado como ayudas a proyectos. En segundo lugar, se ha procedido a la clásica asignación de las dotaciones 
presupuestarias a las ramas de actividad de la $\mathrm{TIO}_{16}(\text { Tabla } 3)^{10}$. Realizada esta doble asignación se observa como los fondos destinados al aumento de renta se concentran en las ramas de Industria manufacturera (25\%), Servicios prestados a las empresas (14\%), Comercio y reparación (13\%) y Hostelería (9\%). Por su parte, los fondos dirigidos a ayudas a proyectos se concentran en las ramas de Construcción (31\%), Maquinaria y material de transporte (27\%), Agricultura $(22 \%)$ y Servicios a las empresas $(16 \%)$. En ambos casos, el crecimiento de la demanda doméstica es mayor que la importada, un $68,61 \%$ para las ayudas a rentas y un $74,83 \%$ para las ayudas destinadas directamente a demanda.

\section{Tabla 3}

Asignación del gasto público del Programa de Desarrollo Rural de Castilla-La Mancha por ramas de actividad (miles de euros)

\begin{tabular}{|l|r|r|r|r|r|c|}
\hline & \multicolumn{3}{|c|}{$\Delta \mathbf{D}$} & \multicolumn{2}{c|}{$\Delta$ RC } \\
\hline & Doméstico & Importado & $\begin{array}{c}\text { Asignación } \\
\text { por ramas }\end{array}$ & Doméstico & Importado & $\begin{array}{c}\text { Asignación } \\
\text { por ramas }\end{array}$ \\
\hline $\begin{array}{l}\text { 1. Agricultura, } \\
\text { Ganadería, caza, } \\
\text { selvicultura y pesca }\end{array}$ & $203.412,07$ & $30.367,13$ & $22 \%$ & $21.276,63$ & $10.986,49$ & $4 \%$ \\
\hline 2. Industrias Extractivas & 0,00 & 0,00 & $0 \%$ & 169,24 & 32,68 & $0 \%$ \\
\hline 3. Energía y Agua & 0,00 & 0,00 & $0 \%$ & $24.075,21$ & $35.154,73$ & $7 \%$ \\
\hline $\begin{array}{l}\text { 4. Industria mafucturera } \\
\text { 5. Maquinaria y material } \\
\text { de transporte }\end{array}$ & $69,910,73$ & $215.739,10$ & $27 \%$ & $6.104,78$ & $62.724,28$ & $9 \%$ \\
\hline 6. Construcción & $331.780,83$ & 0,00 & $31 \%$ & $7.039,86$ & 0,00 & $1 \%$ \\
\hline $\begin{array}{l}\text { 7. Comercio y reparación } \\
\text { 8. Hostelería }\end{array}$ & 0,00 & 0,00 & $0 \%$ & $109.629,35$ & 0,00 & $14 \%$ \\
\hline $\begin{array}{l}\text { 9. Transportes y } \\
\text { comunicaciones }\end{array}$ & $12.542,22$ & 957,78 & $1 \%$ & $24.193,16$ & $6.417,34$ & $4 \%$ \\
\hline $\begin{array}{l}\text { 10. Intermediación } \\
\text { financiera }\end{array}$ & 0,00 & 0,00 & $0 \%$ & $21.864,59$ & $13.252,79$ & $4 \%$ \\
\hline
\end{tabular}

${ }^{10}$ En relación con la regla de reparto existe una propuesta metodológica oficial de la DGXXII (BIPE conseil, 1991) pero, dada su antigüedad y una clasificación que no se adapta a los ejes de intervención establecidos en FEADER, no ha sido de aplicación en el presente trabajo. Se ha considerado más oportuno realizar un reparto teniendo en cuenta los objetivos y acciones financiables en el marco de cada una de las medidas. Esta información viene expresamente recogida en el texto del Programa de Desarrollo Rural de Castilla-La Mancha 2007-2013, así como en el conjunto de Órdenes legislativas de la Junta de Comunidades de Castilla-La Mancha que han regulado la concesión de las ayudas para cada tipo de medida. Toda esta información ha permitido conocer con cierta precisión las intervenciones y conceptos financiables y, por tanto, la diferenciación entre ayudas a rentas y ayudas a proyectos, así como la asignación a las distintas ramas de la tabla input-ouput. 
Tabla 3 (continuación)

Asignación del gasto público del Programa de Desarrollo Rural de Castilla-La Mancha por ramas de actividad (miles de euros)

\begin{tabular}{|c|c|c|c|c|c|c|}
\hline & \multicolumn{3}{|c|}{$\Delta \mathbf{D}$} & \multicolumn{3}{|c|}{$\Delta \mathbf{R C}$} \\
\hline & Doméstico & Importado & $\begin{array}{c}\% \\
\text { Asignación } \\
\text { por ramas }\end{array}$ & Doméstico & Importado & $\begin{array}{c}\% \\
\text { Asignación } \\
\text { por ramas }\end{array}$ \\
\hline $\begin{array}{l}\text { 11. Servicios prestados a } \\
\text { las empresas }\end{array}$ & $154.110,91$ & $18.964,13$ & $16 \%$ & $111.868,14$ & $5.758,02$ & $15 \%$ \\
\hline 12. Administración pública & 0,00 & 0,00 & $0 \%$ & 0,00 & 0,00 & $0 \%$ \\
\hline 13. Educación & $19.100,00$ & 0,00 & $2 \%$ & $5.291,05$ & 0,00 & $1 \%$ \\
\hline $\begin{array}{l}\text { 14. Sanidad y servicios } \\
\text { sociales }\end{array}$ & 0,00 & 0,00 & $0 \%$ & $16.793,99$ & 0,00 & $2 \%$ \\
\hline $\begin{array}{l}\text { 15. Otras actividades } \\
\text { sociales y servicios } \\
\text { personales }\end{array}$ & 0,00 & 0,00 & $0 \%$ & $23.809,95$ & 0,00 & $3 \%$ \\
\hline $\begin{array}{l}\text { Actividades de los } \\
\text { hogares }\end{array}$ & 0,00 & 0,00 & $0 \%$ & $8.286,91$ & 0,00 & $1 \%$ \\
\hline TOTAL & $790.856,76$ & $266.028,15$ & & $545.579,66$ & $249.620,41$ & \\
\hline \% Asignación D - RC & \multicolumn{2}{|c|}{$57,06 \%$} & & \multicolumn{2}{|c|}{$42,94 \%$} & \\
\hline \% Doméstico - Importado & $74,83 \%$ & $25,17 \%$ & & $68,61 \%$ & $31,39 \%$ & \\
\hline
\end{tabular}

\section{RESULTADOS}

\subsection{Impacto sobre la región de Castilla-La Mancha}

El impacto del FEADER sobre la economía regional dependerá de los efectos de arrastre de las distintas ramas a las que finalmente se destinen dichos fondos. Los resultados obtenidos se recogen en la Tabla $4{ }^{11}$.

En relación con el gasto público destinado a ayudas a proyectos, el Programa de Desarrollo Rural de Castilla-La Mancha se prevé que incremente la producción regional en 1.119,5 millones de euros, el valor añadido en 520,1 millones de euros y el número de trabajadores en 14.554. El cociente entre la producción generada y los fondos gastados determina un multiplicador de 1,49; es decir, por cada euro gastado en la región se aumenta el output por valor de 1,49. Si nos fijamos en el gasto público destinado a las ayudas a rentas, el

${ }^{11}$ Los resultados obtenidos se basan en la presunción de la ejecución completa del cuadro financiero. Es razonable pensar que, en el actual contexto de crisis económica, la necesaria cofinanciación por parte de los beneficiarios finales en determinadas medidas podría hacer peligrar la ejecución completa de los fondos asignados. No obstante, la posibilidad de introducir modificaciones entre medidas que recoge el propio Reglamento (CE) 1698/2005 y, lo que es más importante, el interés de la administración autonómica en no "perder fondos" minimiza la probabilidad de incumplimientos en la ejecución. 
impacto sobre la producción de la región objetivo se ha estimado en 671,7 millones de euros, el del valor añadido en 330,9 millones de euros y el del empleo en 10.599 trabajadores, siendo 1,23 el valor del multiplicador. Se observa, pues, que la capacidad de arrastre de las ayudas a rentas es inferior a la de las ayudas a proyectos, lo que se explica por la distinta composición sectorial del gasto y porque los fondos destinados a mantener las rentas se gastan en bienes y servicios con una mayor propensión a importar. En cifras globales, considerando la inversión total prevista del FEADER, el impacto calculado supondrá un aumento del 3,03\% de la producción de la región, un 3,20\% del valor añadido y un $3,40 \%$ del nivel de empleo. En valores anualizados, la producción aumentará un $0,43 \%$ cada año.

\section{Tabla 4}

Impacto del FEADER sobre producción, valor añadido y empleo. Productividad total y aparente. (Miles de euros y número de personas)

\begin{tabular}{|c|c|c|c|c|c|c|c|c|c|c|}
\hline & \multicolumn{4}{|c|}{$\Delta \mathbf{d}$} & \multicolumn{4}{|c|}{$\Delta \mathrm{rc}$} & \multicolumn{2}{|c|}{$\Delta$ Total } \\
\hline & CLM & M Esp & $M U E$ & M RM & CLM & M Esp & M UE & M RM & CLM & M Total \\
\hline FEADER & 799.339 & 217.410 & 25.542 & 14.594 & 545.580 & 199.623 & 32.116 & 17.881 & 1.344 .919 & 507.166 \\
\hline$\Delta \mathrm{X}$ con MRIO & 1.195 .692 & 1.130 .276 & 118.715 & 64.872 & 671.735 & 813.042 & 98.746 & 71.349 & 1.867 .427 & 2.297 .000 \\
\hline$\triangle$ VA con MRIO & 520.108 & 397.918 & 45.505 & 26.760 & 330.952 & 293.954 & 38.743 & 30.812 & 851.060 & 833.693 \\
\hline$\Delta \mathrm{E}$ & 14.554 & 9108 & 977 & 539 & 10.599 & 6.092 & 795 & 549 & 25.153 & 18.061 \\
\hline $\begin{array}{l}\text { Productividad } \\
\text { total: (X/E) } \\
\end{array}$ & 82 & 124 & 121 & 120 & 63 & 133 & 124 & 130 & 74 & 127 \\
\hline $\begin{array}{l}\text { Productividad } \\
\text { aparente: (VA/E) }\end{array}$ & 36 & 44 & 47 & 50 & 31 & 48 & 49 & 56 & 34 & 46 \\
\hline
\end{tabular}

Nota: Para obtener el empleo generado en la UE y en el resto del mundo se ha utilizado el coeficiente de empleo directo de España, al no disponer de información de empleo de esas regiones.

\subsection{Impacto sobre España y el resto del mundo}

Como ya se ha señalado, los efectos del Programa de Desarrollo Rural de Castilla-La Mancha sobrepasan las fronteras de la región a través de las importaciones directas e indirectas necesarias para satisfacer los incrementos de producción y de renta que generan las ayudas. Los resultados obtenidos indican que la repercusión sobre el output será mayor fuera de la región de Castilla-La Mancha que dentro de ella. En concreto, se calcula un incremento de 2.297 millones de euros para España, la Unión Europea y el resto del mundo y de 1.867 millones de euros para la región manchega (Tabla 4) ${ }^{12}$. Así pues, parte de

${ }^{12}$ Esta distribución es superior a la encontrada por Pérez, Donés y Llano (2009) para los fondos de cohesión recibidos por las regiones españolas entre 1995-1999, en los que el efecto multiplicador que quedaba en la región objetivo alcanzaba de media el 63,9\%. 
los fondos recibidos acaban repercutiendo fuera de la región objetivo ${ }^{13}$; si bien, es previsible que beneficien principalmente a España, dada la importancia del factor proximidad para la generación de spillovers interregionales derivados del previsible desarrollo del capital físico, tecnológico y social de la región objetivo.

Del crecimiento total de la producción generado, un $44,8 \%$ se daría en Castilla-La Mancha, un 46,7\% en el resto de España, un 5,2\% en la Unión Europea y un $3,3 \%$ en el resto del mundo ${ }^{14}$. Una vez que las mercancías son importadas de otros países, su importancia relativa sobre la producción es sólo algo superior en la Unión Europea frente al resto del mundo.

Como otros territorios, la región de Castilla-La Mancha se encuentra en un contexto de creciente globalización. Ya desde el año 2005 es manifiesta la tendencia al predominio del offshoring hacia países con menor coste laboral como forma de hacer frente a un contexto internacional de reducción de precios y salarios (Cadarso et al., 2008). Es decir, una vez que eliminamos las ventajas que reporta la proximidad física y cultural que supone comerciar con regiones de un mismo país, los costes de transacción no parecen ser muy distintos para las importaciones internacionales de las nacionales, lo que se ha de considerar en términos de estrategia de política comunitaria, ya que los efectos fuera de la Unión Europea pueden ser incluso crecientes en el futuro.

En relación con el valor añadido, los efectos de la intervención del FEADER son algo mayores en la región que fuera de ella; en concreto, 851 millones de euros frente a 833. Por último, el impacto sobre el empleo es también superior a nivel interno, al generarse 25.153 puestos de trabajo en Castilla-La Mancha y 18.061 fuera de ella (15.200 en España, 1.772 en la Unión Europea y 1.088 en el resto del mundo) ${ }^{15}$. El cambio de la importancia del efecto, según se considere la producción, el valor añadido o el empleo, viene dado por la diferente composición sectorial de los gastos, así como por las distintas tecnologías de producción existentes en la economía castellano-manchega, española, comunitaria y del resto del mundo. Las mercancías producidas en Castilla-La Mancha mantienen una mayor intensidad en empleo, o son menos

${ }^{13}$ Esto es especialmente significativo en el caso de las regiones de menor renta, como Castilla-La Mancha, Extremadura y Andalucía, que no son capaces de apropiarse de forma significativa del comercio interregional que generan los fondos de cohesión (Pérez, Dones y Llano, 2009).

${ }^{14}$ En este punto cabe recordar la posible sobrestimación de datos para España, pues consideramos que no importa bienes para atender el incremento de la demanda de Castilla-La Mancha. A nivel agregado no supondría cambios, pues el efecto multiplicador calculado sobre las regiones no objetivo es correcto. La única diferencia la encontraríamos en el impacto exacto acontecido en España, que debería ser inferior, a favor del impacto en la Unión Europea y en el resto del mundo.

${ }^{15}$ Consideramos el coeficiente directo de empleo de la Unión Euroepa y del resto del mundo igual al de España. 
productivas, por lo que requieren una mayor cantidad de trabajadores por unidad monetaria producida. Los resultados recogidos en la Tabla 4 indican que la productividad del trabajo total de Castilla-La Mancha es de 74 (miles de euros por trabajador) y la aparente de 34 , datos sensiblemente inferiores a los obtenidos para España, la Unión Europea y el resto del mundo -valores que oscilan entre 120 y 133 para la productividad total, y entre 44 y 46 para la productividad aparente. Estos diferenciales territoriales pueden deberse, tal y como se recoge en Peña (2007), a tres posibles elementos: a la composición de las economías regionales, a los procesos de reestructuración de las mismas y a la difusión geográfica de las innovaciones.

En relación con la diferenciación entre las ayudas a rentas y a proyectos, los datos muestran que el mayor impacto se produce en el segundo tipo, si bien el estudio de las productividades matiza los resultados obtenidos en términos de empleo. La Tabla 4 pone de relieve que las productividades vinculadas a las ayudas a rentas son mayores que las destinadas a proyectos, con lo que el aumento del empleo será menor en el primer tipo de fondos que en el segundo.

\subsection{Efectos sectoriales}

A la hora de analizar los efectos sectoriales del Programa de Desarrollo Rural de Castilla-La Mancha procederemos, en primer lugar, a comentar los resultados diferenciando entre las cuatro áreas geográficas consideradas en el estudio: Castilla-La Mancha, España, Unión Europea y resto del mundo. Posteriormente, nos centraremos en los resultados de la región objetivo: Castilla-La Mancha.

En el caso de los fondos destinados a las ayudas a proyectos, se observa que en la región objetivo predominan los efectos sobre las ramas de la Construcción ( $42 \%$ del total), la Agricultura, la Industria manufacturera y los Servicios a empresas (Gráfico 1). El impacto de estos fondos sobre las importaciones es sensiblemente distinto, pues se concentra en las de la Industria manufacturera, Maquinaria y Servicios a las empresas. Estas importaciones son significativas tanto en el comercio con el resto de regiones españolas, como con los países de la Unión Europea y del resto del mundo.

En las ayudas directas a rentas, el impacto recae principalmente en las ramas de Servicios a las empresas, Industria manufacturera, Comercio y reparación y Hostelería (Gráfico 2). A nivel nacional, las ramas más beneficiadas son las mismas pero añadiendo la de Industrias extractivas. La ausencia de una importante industria de media y alta tecnología en Castilla-La Mancha le obliga a importar una gran cantidad de bienes intermedios y finales para satisfacer los incrementos de demanda final. Por otro lado, llama la atención que el montante total de importaciones de Servicios a las empresas sean tan importantes como el consumo doméstico de éstos. El desarrollo de las tecnologías de la información 
y las comunicaciones y la existencia de economías de aglomeración en comunidades autónomas próximas (Madrid y Comunidad Valenciana) permite que sean prestados desde el exterior.

\section{Gráfico 1}

Impacto del Programa de Desarrollo Rural de Castilla-La Mancha (Ayudas a proyectos) sobre la Producción distinguiendo por área geográfica (Miles de Euros)

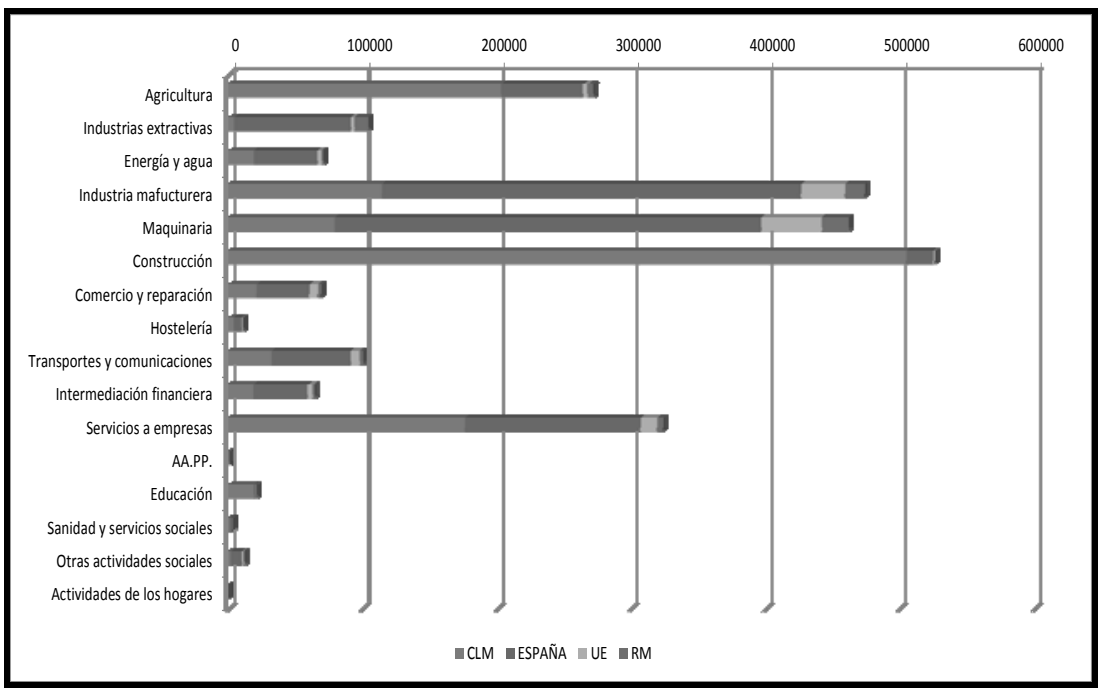

\section{Gráfico 2}

Impacto del Programa de Desarrollo Rural de Castilla-La Mancha (Transferencia de rentas) sobre la producción distinguiendo por área geográfica (Miles de Euros)

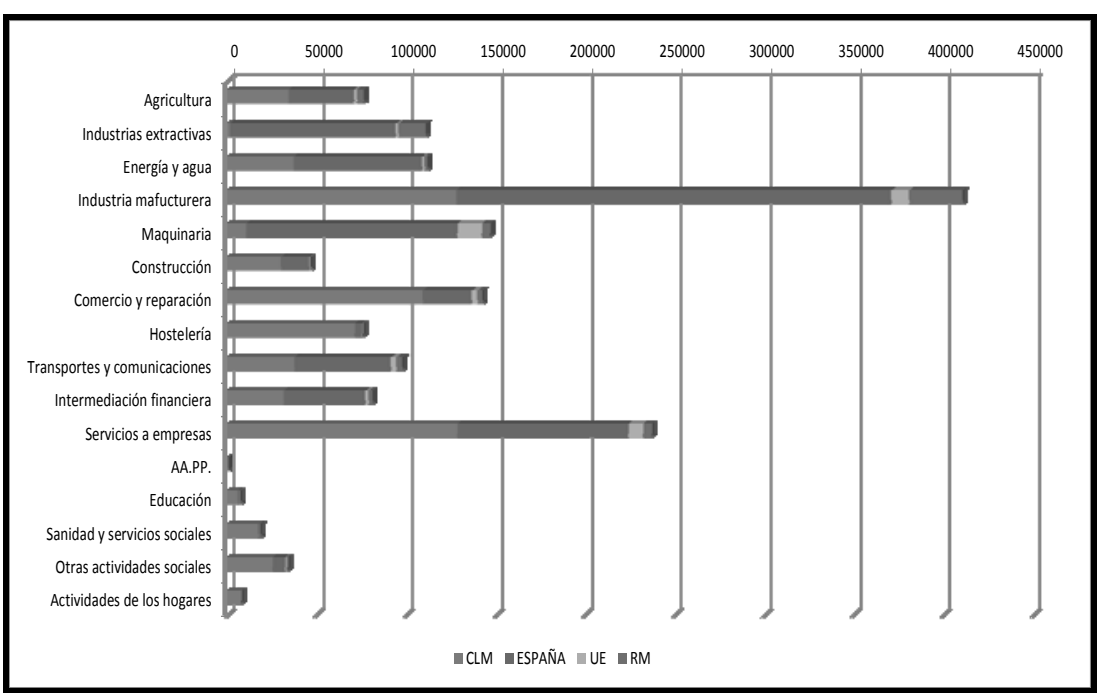


Pasamos a continuación a realizar el análisis sectorial de los impactos sobre la región de Castilla-La Mancha.

En relación con las ayudas a proyectos, y atendiendo a los efectos generados dentro de la región objetivo, el mayor impacto sectorial sobre la producción recae en las ramas de Agricultura (6,19\%), Construcción (5,55\%), Maquinaria $(4,82 \%)$ y Servicios a empresas $(3,27 \%)$ (Gráfico 3). Estas ramas coinciden, mayoritariamente, con aquellas a las que se asignaron primariamente los fondos. Puede observarse también crecimiento en ramas como las de Intermediación financiera $(1,26 \%)$ e Industrias extractivas $(1,11 \%)$ que no han percibido directamente ayudas pero que se benefician del efecto arrastre de las ramas receptoras.

\section{Gráfico 3}

Impacto del Programa de Desarrollo Rural de Castilla-La Mancha (Ayudas a proyectos) sobre la producción por ramas de actividad en la región de Castilla-La Mancha

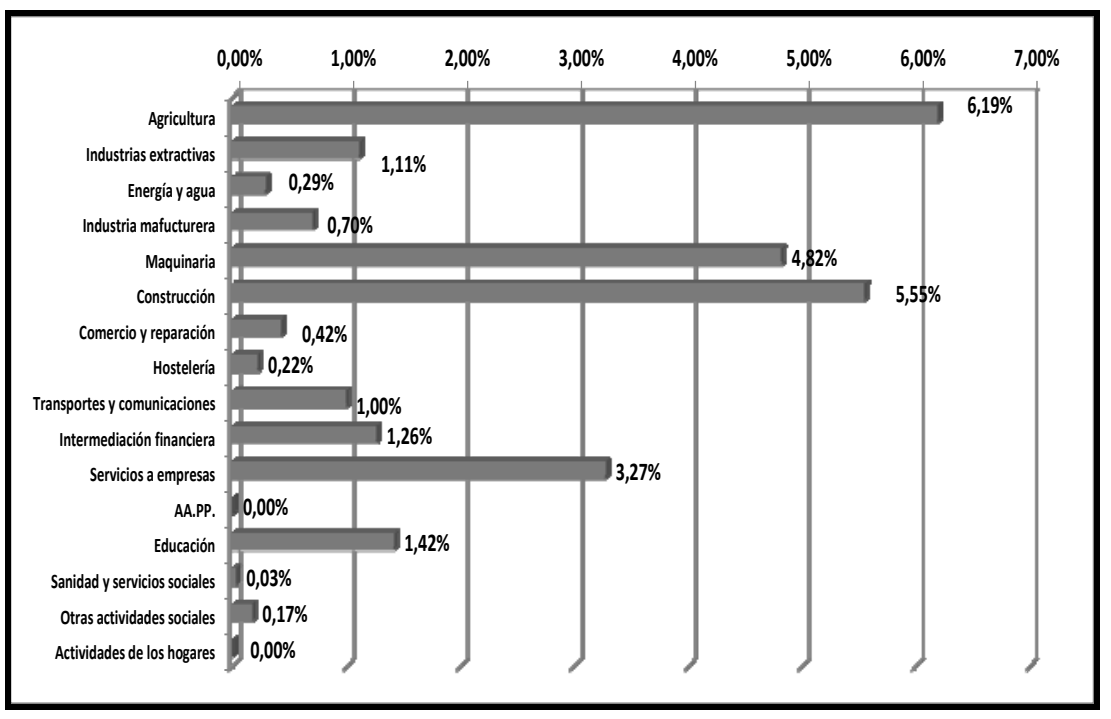

Por su parte, en el caso de las ayudas directas a rentas, los mayores impactos sobre la producción se concentran en las ramas de Actividades de los hogares $(4,73 \%)$, Hostelería $(4,20 \%)$, Otras actividades sociales $(2,48 \%)$, Servicios a empresas $(2,39 \%)$, Comercio y reparación $(2,20 \%)$ e Intermediación financiera $(2,14 \%)$. En términos porcentuales el mayor crecimiento recae en actividades relativas a los servicios con baja propensión a importar (excepto Servicios a empresas). Los fondos destinados a la mejora de las rentas tienen la capacidad de vertebrar dentro de la región un sector servicios que responde a las necesidades sociales y productivas del entorno (Gráfico 4). 


\section{Gráfico 4}

Impacto del Programa de Desarrollo Rural de Castilla-La Mancha (Transferencia de rentas) sobre la producción por rama de actividad en la región de Castilla-La Mancha

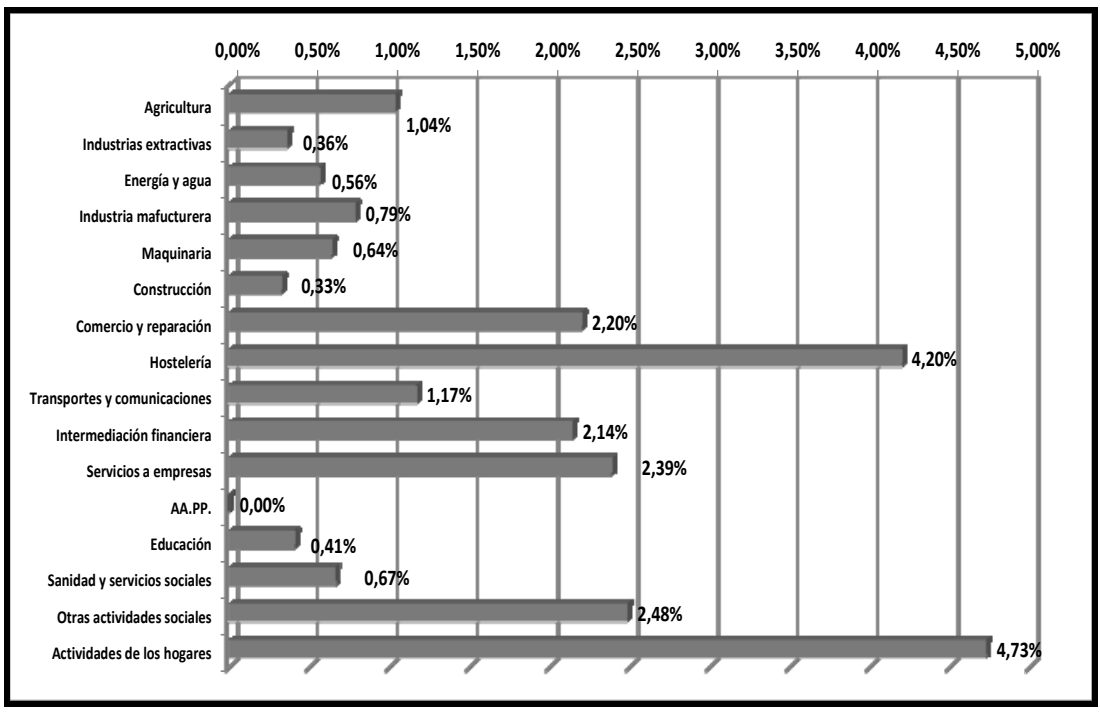

\section{CONCLUSIONES}

A la hora de abordar la evaluación de impacto del Fondo Europeo Agrícola de Desarrollo Rural, la metodología Input-Output presenta ventajas importantes relacionadas con la gran cantidad de información generada sobre la eficacia del gasto. Además, la desagregación de la información permite aproximarse sectorialmente a los efectos de dicho Fondo y cómo éstos se distribuyen por toda la economía.

Desde el punto de vista metodológico, el presente estudio presenta un modelo input-output multiregional que permite calcular los impactos de los programas de desarrollo rural tanto en la región objetivo como fuera de ella. Por otra parte, al diferenciarse entre los fondos destinados a ayudas directas a rentas (aumento del consumo) de los fondos asignados a ayudas a las iniciativas o proyectos de desarrollo rural (aumento de la demanda final) puede compararse la mayor o menor eficacia de unos y otros, así como los efectos a nivel sectorial.

Desde el punto de vista empírico los resultados obtenidos confirman la importancia que el Programa de Desarrollo Rural de Castilla-La Mancha tendrá sobre la economía regional durante el período 2007-2013. Concretamente, se ha estimado en un $3,03 \%$ el efecto sobre la producción $(0,43 \%$ de incremento anual), un $3,20 \%$ sobre el valor añadido, y la creación de 25.153 empleos, que suponen un incremento del 3,40\%, para el periodo en su conjunto. El estudio de los efectos fuera de la región objetivo muestra resultados relevantes a nivel de 
producción. Los incrementos del output en España, Unión Europea y resto del mundo atribuibles a la financiación del FEADER en Castilla-La Mancha, son un $23 \%$ superior a los generados dentro de la región objetivo. Este resultado refleja la fuerte apertura exterior de la economía castellano-manchega, lo que hace que una gran parte del efecto multiplicador se derive hacia otras regiones y al extranjero, motivado por la importación de bienes finales e intermedios necesarios para atender el incremento de la demanda interna. En términos de valor añadido y empleo los efectos fuera de Castilla-La Mancha son algo menores, debido principalmente a la distinta composición sectorial de los gastos y a la menor productividad observada en la región manchega. En este punto cabe resaltar que la concesión de fondos a determinadas regiones no sólo va a generar efectos positivos sobre las mismas, sino que éstos se van a extender por todas aquellas otras con las que mantienen lazos comerciales. Esta pérdida de efecto multiplicador en las regiones objetivo, dependerá del grado de apertura de las mismas y de su capacidad sectorial para atender los aumentos de demanda generados. Cuanto mayor sea esta capacidad, mayores serán también los efectos que se generen dentro de la propia región, logrando con ello un mayor grado de éxito de este tipo de planes.

A nivel sectorial observamos cómo, tanto para el caso de las ayudas destinadas a la inversión en proyectos como para las dedicadas a incrementos de renta, el efecto fuera de Castilla-La Mancha recae en buena medida sobre ramas de media y alta tecnología, de las cuales adolece la economía castellanomanchega. La diferenciación entre los dos tipos de ayuda permite analizar con más detalle sobre qué ramas productivas recaen los mayores efectos de arrastre. En el caso de las primeras, el mayor impacto se produce en las ramas de Construcción, Agricultura y Servicios a empresas; en las ayudas a rentas los mayores efectos recaen sobre las ramas de Industria manufacturera, Servicios a empresas, Comercio y reparación y Hostelería.

Como consideración final cabe añadir que, dada la dimensión cualitativa de los objetivos perseguidos por los Programas de Desarrollo Rural (dinamización socio-económica del territorio y mejora de la calidad de vida), una evaluación de tipo cuantitativo, complementa pero no sustituye a las necesarias evaluaciones cualitativas; de hecho, la complementariedad de ambas metodologías permitirá un conocimiento más preciso de la eficacia del FEADER y, en su caso, una mayor justificación ante la sociedad de dichas inversiones. 


\section{REFERENCIAS BIBLIOGRÁFICAS}

ACQUAYE, A. A., y DUFFY, A. P. (2010). "Input - output analysis of Irish construction sector greenhouse gas emissions ". Building and Environment, 45, pp. 784 - 791.

ANDREW, R., PETERS, G. P., y LENNOX, J. (2009). "Approximation and Regional Aggregation in Multi-regional Input-Output Analysis for National Carbon Footprint Accounting". Economic Systems Research, 21(3), pp. 311-335.

CADARSO, M. A., GOMEZ, N., LOPEZ, L. A., y TOBARRA, M. A. (2008). "The EU Enlargement and the Impact of Outsourcing on Industrial Employment in Spain, 1993-2003". Structural Change and Economic Dynamics, 19(1), pp. 95-108.

CÁMARA, A., y MARCOS, M. A. (2009). "Análisis del impacto de los Fondos Europeos 2000-2006 en la Comunidad de Madrid a partir de la matriz de contabilidad social del año 2000". Investigaciones regionales, (16), pp. 7192.

CARDENETE, M. A., y LLOP, M. (2005). "Modelos multisectoriales de equilibrio general aplicado en España: Una revisión.". Estudios de Economia Aplicada, 23(2), pp. 385-404.

COLINO, J., y MARTÍNEZ, J. M. (2005). "El Desarrollo rural: segundo pilar de la PAC". En J. L. García Delgado y M. D. Garcia Grande, (eds.): Política agraria común: balance y perspectivas. (pp.). La Caixa, pp. 70-99

COMISIÓN EUROPEA. (1989): El futuro del mundo rural [Texto impreso] : comunicación de la Comisión al Consejo y al Parlamento : documento 7957/88 (agosto de 1988). Madrid: Servicio de Extensión Agraria, Ministerio de Agricultura.

COMISIÓN EUROPEA. (2005a). "Reglamento (CE) 1290/2005 sobre la financiación de la política agrícola común".

COMISIÓN EUROPEA. (2005b). "Reglamento (CE) 1698/2005 relativo a la ayuda al desarrollo rural a través del Fondo Europeo Agrícola de Desarrollo Rural (FEADER)".

JUNTA DE COMUNIDADES DE CASTILLA-LA MANCHA. (2009): Programa de desarrollo rural de Castilla-la Mancha 2007-2013. Toledo: Consejería de Agricultura y Medio Ambiente.

LIMA, M. C., y CARDENETE, M. A. (2005). "Análisis de impacto de los fondos FEDER recibidos por una economía Regional: un enfoque a través de Matrices de Contabilidad Social". Presupuesto y gasto público, 40(3), pp. 113-132.

LLANO, C. (2004). "The interregional trade in the context of a multirregional input-output model for Spain". Estudios de economía aplicada, 22(3), pp. 539-576. 
MARÍN, A., y PARDO, A. (2009). "El impacto de los fondos estructurales 20002006 sobre la región de Castilla y León: Un análisis input-output". Papeles de Economía Española, (123), pp. 157-177.

MURILLO, E. (2007): Análisis de los efectos económicos de la política regional europea en Andalucía. Sevilla: CES.

PEÑA, A. R. (2007). "Análisis sectorial de la productividad y de la estructura productiva en Andalucía". Estudios de Economia Aplicada, 25(3), pp. 691726.

PEREZ, J., DONES, M., y LLANO, C. (2009). "An Interregional Impact Analysis of the EU Structural Funds in Spain (1995-1999)". Papers in Regional Science, 88(3), pp. 509-529.

PETERS, G. P., y HERTWICH, E. G. (2006). "Pollution embodied in trade: The Norwegian case". Global Environmental Change, 16(4), pp. 379-387.

RAY, C. (2000). "Endogenous socio-economic development in the European Union -- issues of evaluation". Journal of Rural Studies, 16(4), pp. 447-458. 
